\title{
Long non-coding RNA expression profile in vulvar squamous cell carcinoma and its clinical significance
}

\author{
SHA NI, XIAOYU ZHAO and LING OUYANG \\ Department of Gynecology and Obstetrics, Shengjing Hospital Affiliated to China Medical University, \\ Heping, Shenyang, Liaoning 110004, P.R. China
}

Received February 12, 2016; Accepted March 21, 2016

DOI: $10.3892 /$ or.2016.5075

\begin{abstract}
Researchers have recently demonstrated the key role of long non-coding RNAs (lncRNAs) in regulating embryogenesis and gene expression. However, the exact mechanism used by lncRNAs in carcinogenesis is still unclear. In particular, studies regarding the role of lncRNAs in vulvar squamous cell carcinomas (VSCCs) are limited. Using microarray analysis, the genome-wide expression profile of lncRNAs was investigated in four paired VSCCs and adjacent normal vulvar tissues. Accordingly, several novel lncRNA candidates (HOAIR, MALAT1, MEG3, NEAT1, MIR31HG and LINC00478) were chosen for further study and real-time reverse transcription PCR (RT-PCR) was used to confirm the expression levels among 35 tissue samples. A panel of dysregulated lncRNAs (MEG3 and MALAT1) were also identified as potential biomarkers as they also correlated with VSCC carcinogenesis. In summary, the results revealed that aberrantly expressed lncRNAs may be a factor in VSCC pathogenesis, potentially providing new biomarkers and therapeutic targets for VSCC.
\end{abstract}

\section{Introduction}

Vulvar squamous cell carcinoma (VSCC) is a relatively rare malignant tumor in women which accounts for $80-90 \%$ of female genital tract cancers (1). Early stage VSCC is primarily treated with surgery, however, postoperative complications can occur. Several of these complications, such as lower limb lymphatic obstruction and vulvar morphologic changes, can have a deep impact on the quality of life of patients. The treatment for advanced vulvar cancer includes radiotherapy. The survival rate for patients with advanced VSCC is only $30 \%$ due to the reduced tolerance of vulvar tissues to radiation.

Correspondence to: Dr Ling Ouyang, Department of Gynecology and Obstetrics, Shengjing Hospital Affiliated to China Medical University, 36 Sanhao Street, Heping, Shenyang, Liaoning 110004, P.R. China

E-mail: ouyangling1964@gmail.com

Key words: long non-coding RNA, vulvar squamous cell carcinomas, microarray, HOTAIR, MALAT1
Thus, it is important to better understand the pathogenesis of VSCC and to identify possible effective biomarkers to aid diagnosis.

Over the past decade, research into the non-coding RNA (ncRNA) domain has attracted significant attention. Long non-coding RNAs (lncRNAs) >200 nucleotides in length do not encode any proteins $(2,3)$ but are involved in regulating gene functions at transcriptional and post-transcriptional levels (4). These gene functions include epigenetic regulation, chromatin modification, cell cycle regulation, nuclear trafficking, transcription and splicing (5-8). The dysregulation of lncRNAs is linked to various human diseases, particularly cancer (9-11).

In the present study, an lncRNA expression profile was established from samples obtained from four paired VSCC subjects and their adjacent non-tumor (NT) tissues through a microarray platform. The aim of the present study was to identify dysregulated lncRNAs and mRNAs in VSCC patients. Six of these lncRNAs were evaluated by real-time reverse transcription-polymerase chain reaction (RT-PCR) in 35 cases with benign vulvar diseases, VSCC and their pericarcinoma tissues according to the top 30 upregulated and top 30 downregulated IncRNAs.

\section{Materials and methods}

Patients and sample collection. The present study protocol was approved by the Institutional Review Board of Shengjing Hospital Affiliated to China Medical University. Written informed consent was obtained from all patients. A total of 16 VSCC patients, diagnosed with VSCC between January 2013 and June 2015, were enrolled in the present study. Adjacent tissues in 4 VSCC patients, 7 cases of vulvar lichen sclerosus, 6 cases of vulvar leukoplakia and 2 cases of vulvar intraepithelial neoplasia (VIN) grade I were obtained as the control group. The clinical characteristics of the patients and their tumors are summarized in Table I. None of the patients had received prior radiotherapy or other anticancer treatment. All histopathological samples were obtained at surgery and were confirmed as VSCC. All samples were stored at $-80^{\circ} \mathrm{C}$ until use.

Total RNA extraction. Total RNA was isolated from the frozen samples using TRIzol reagent (Invitrogen, Carlsbad, CA, USA), 
Table I. Relationship between the expression of NEAT1, LINC00478 or MIR31HG and the clinicopathological features of the patients with VSCC.

\begin{tabular}{lcccc}
\hline & No. of patients $(\%)$ & NEAT1 & LINC00478 & MIR31HG \\
\hline Age (years) & & & & \\
$\leq 63$ & $8(50)$ & $2.59(1.23,2.90)$ & $8.44(5.06,7.09)$ & $9.09(6.61,13.67)$ \\
$>63$ & $8(50)$ & $4.09(1.86,7.68)$ & $8.93(8.35,10.45)$ & $8.91(7.67,10.04)$ \\
P-value & & $\mathbf{0 . 2 7}$ & $\mathbf{0 . 0 1 2}$ & $\mathbf{1 . 0 0 0}$ \\
FIGO Stage & & & \\
I & $7(43.8)$ & $3.40(1.49,8.28)$ & $8.33(5.55,9.32)$ & $10.08(6.46,14.49)$ \\
II & $1(6.3)$ & & & \\
III & $8(50)$ & $2.59(1.23,2.90)$ & $6.68(5.26,10.02)$ & $8.91(7.67,10.04)$ \\
IV & $0(0)$ & & & $\mathbf{1 . 0 0 0}$ \\
P-value & & $\mathbf{0 . 0 8}$ & & $10.08(9.62,15.58)$ \\
Tumor differentitation & $9(56.3)$ & $4.09(1.86,7.68)$ & $9.32(8.36,11.43)$ & $7.08(6.26,7.90)$ \\
Well & $5(31.3)$ & $2.71(1.91,3.00)$ & $5.55(5.28,6.68)$ & $7.64(6.74)$ \\
Moderate & $2(12.5)$ & $1.41(0.23)$ & $4.74(4.47)$ & $\mathbf{0 . 0 0 7}$ \\
Poor & & $\mathbf{0 . 2 1 4}$ & $\mathbf{0 . 0 0 3}$ & $8.91(6.92,12.02)$ \\
P-value & & & & $9.14(7.28,11.72)$ \\
Lymphatic metastasis & $10(60)$ & $3.22(2.90,6.46)$ & $8.93(8.33,10.22)$ & $\mathbf{1 . 0 0 0}$ \\
N0 & $6(37.5)$ & $1.34(0.97,1.77)$ & $5.71(5.17,9.32)$ & $\mathbf{0 . 0 6 5}$ \\
N1, N2, N3 & & $\mathbf{0 . 0 0 2}$ & & \\
P-value & & &
\end{tabular}

VSCC, vulvar squamous cell carcinoma; FIGO, International Federation of Gynecology and Obstetrics.

according to the manufacturer's protocols. All samples were quantified using a NanoDrop ND-1000 spectrophotometer (Thermo Fisher Scientific, Waltham, MA. USA). The purity of total RNA was examined by its absorbance ratio at 260-280 $\mathrm{nm}$. The absorbance ratios at $\mathrm{A}_{260 / 280}$ were between 1.8 and 2.0 .

IncRNA and mRNA microarray analysis. Four paired samples were chosen for the experiments performed at CapitalBio Corporation Laboratories (CapitalBio Corp., Beijing, China). Generally, total RNA which was extracted using TRIzol reagent for microarray was reverse-transcripted to double-stranded complementary DNA (cDNA). cDNA synthesis, purification, labeling and hybridization were carried out according to the manufacturer's instructions. IncRNA expression profiling was performed using lncRNAs and mRNA 4x180K Human Gene Expression Microarray V4.0 (CapitalBio Corp.). The data extracted were analyzed by Agilent Feature Extraction version 10.7 (Agilent Technologies, Santa Clara, CA, USA), and were summarized and normalized using Agilent GeneSpring software version 11.5. Differentially expressed lncRNAs and mRNAs were identified through an absolute fold-change $>2$ at a $\mathrm{p}<0.05$.

Functional analysis. Gene ontology (GO) and pathway analyses were performed in order to better understand the functions of differentially expressed lncRNAs and mRNAs in VSCC. GO terms consisted of biological processes, cellular components, and molecular functions that were used to annotate and classify gene function. Pathway analysis was based on the Kyoto Encyclopedia of Genes and Genomes (KEGG) database that place differentially expressed mRNAs. In brief, the Fisher's exact test and t-test were used. A $p<0.05$ was considered to indicate a statistically significant result.

IncRNA-mRNA co-expression network analysis. A correlation analysis was constructed between differentially expressed lncRNAs and mRNA by calculating the Pearson correlation coefficients for each dysregulated lncRNA and mRNA. A significant correlation was defined as a correlation $>0.99$ or $\leq 0.99$ at a $\mathrm{p}<0.05$.

Real-time PCR. Single-stranded cDNA was reverse transcribed using the PrimeScript RT reagent kit with gDNA Eraser Perfect Real-Time (Takara Bio, Kyoto, Japan), according to the manufacturer's instructions. Real-time PCR was performed using SYBR Premix Ex Taq Tli RNase H Plus (Takara Bio) and a Roche LightCycler 480 II system (Roche, Basel, Switzerland). The $20 \mu \mathrm{l}$ real-time PCR reaction mixture contained $3 \mu \mathrm{l}$ cDNA, $10 \mu$ l SYBR Premix Ex Taq, $4 \mu$ l RNase-Free Water, and $1.5 \mu \mathrm{l}$, each, of forward and reverse primers. The PCR conditions were as follows: $30 \mathrm{sec}$ at $95^{\circ} \mathrm{C}$, followed by 40 cycles at $95^{\circ} \mathrm{C}$ for $5 \mathrm{sec}$ and at $60^{\circ} \mathrm{C}$ for $30 \mathrm{sec}$. All samples were run in triplicate for analysis. Glyceraldehyde-3-phosphate dehydrogenase (GAPDH) was used as an endogenous control. The relative abundance of lncRNA expression was calculated using the threshold cycle $(\mathrm{Ct})$ method with relative quantitation (12). The primer sequences were designed in the laboratory 
Table II. RT-PCR primers for lncRNA expression analysis.

\begin{tabular}{ll}
\hline Target name & \multicolumn{1}{c}{ Primer sequence (5'-3') } \\
\hline $\begin{array}{l}\text { MIR31HG-Forward } \\
\text { MIR31HG-Reverse }\end{array}$ & CAGGTCTCCAGGTGTTCCAG \\
LINC00478-Forward & AAGATGACAAGAGCACCTCAAAG \\
LINC00478-Reverse & GACCTCAGCCTCCTCCATTA \\
MEG3-Forward & GCTGCCCATCTACACCTCA \\
MEG3-1-Reverse & CCTCTTCATCCTTTGCCATC \\
NEAT1-Forward & GATGCGCGCCTGGGTGTAGTT \\
NEAT1-Reverse & CATGCAGCCTGCCCCACTGT \\
MALAT1-Forward & CCGAGCTGTGCGGTAGGCATT \\
MALAT1-Reverse & CGGTTTCCTCAAGCTCCGCCT \\
HOTAIR-Forward & GGTCCTGCTCCGCTTCGCAG \\
HOTAIR-Reverse & ACGCCCCTCCTTCCTCTCGC \\
GAPDH-Forward & ACCCACTCCTCCACCTTTGAC \\
GAPDH-Reverse & TGTTGCTGTAGCCAAATTCGT
\end{tabular}

RT-PCR, real-time reverse transcription PCR; lncRNA, long non-coding RNA.

and were synthesized by Sangon Biotech (Sangon, Shanghai, China), and are shown in Table II.

Statistical analysis. Statistical analysis was performed using Social Sciences (SPSS) 19.0 software (SPSS, Inc., Chicago, IL, USA). The independent-sample t-test was used to evaluate the expression levels of lncRNAs. Association between lncRNAs and clinicopathological factors was analyzed with the Mann-Whitney U test which was used for comparison between the two groups and the Kruskal-Wallis test which was used for comparison among the three groups. A p $<0.05$ (two-tailed) was regarded as statistically significant.

\section{Results}

Expression profiles of lncRNAs in VSCC tissues. From the lncRNA gene expression, dysregulated lncRNAs were identified in four paired vulvar squamous cell tumor tissues and the adjacent normal vulvar tissue samples. Among the 15,840 lncRNA transcripts analyzed, 312 lncRNAs were observed as upregulated (fold-change, $>2 ; \mathrm{p}<0.05$ ) whereas 1,469 lncRNAs were detected as downregulated (fold-change $>2$; $\mathrm{p}<0.05$ ) in the VSCC samples. Of these, AI769947 (foldchange, 34.2) was the most highly upregulated lncRNA in the VSCC tissues. FER1L4 (fold-change, 48.9) was the most extensively downregulated lncRNA. Hierarchical clustering analysis was performed to determine whether the expression patterns in the VSCC tissues were significantly different from these patterns in the adjacent normal tissues (Fig. 1A).

Overview of the mRNA profile in VSCC. Up to 33,045 coding transcripts were detected in the VSCC tissues and the NTs, of which 21,788 showed significantly differential expression (fold-change, $>2$; $\mathrm{p}<0.05$ ). Among them, 1,521 were upregu-
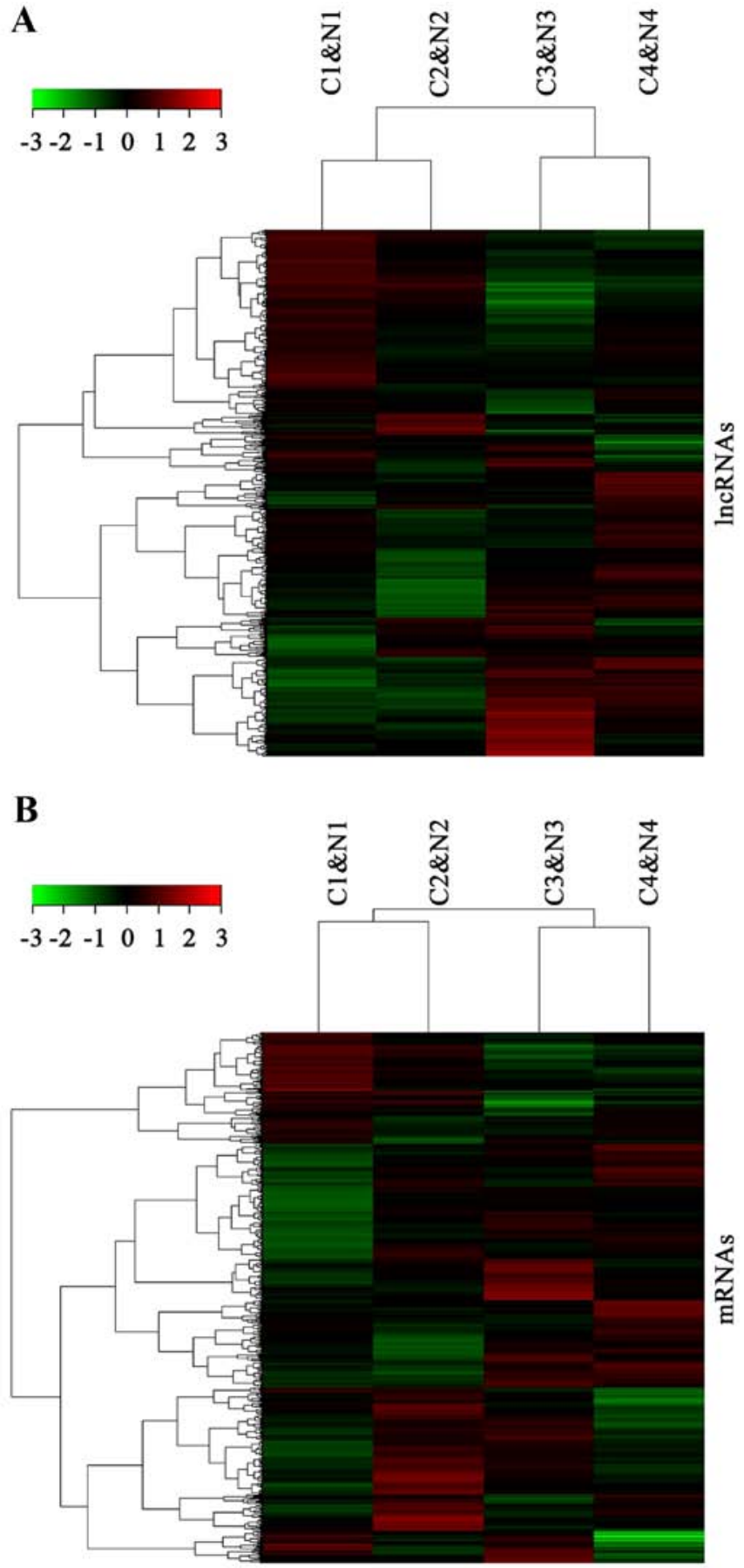

Figure 1. Heat map showing differentially expressed (A) lncRNAs and (B) mRNAs between VSCC and NT samples (fold-change, $>2.0 ; \mathrm{p}<0.05$ ). Each row indicates one non-coding or coding RNA and each column represents one tissue sample. Red indicates high relative regulation and green indicates low relative downregulation.

lated and 4,694 were downregulated in the VSCC tissues. Their distinct expression patterns were evaluated by hierarchical clustering analysis (Fig. 1B).

GO and pathway analysis. Go analysis (comprised of biological processes, cellular components and molecular functions) was performed in order to determine the genes and gene product enrichment. Through GO analysis, the significant differentially expressed mRNAs were principally associated 
A
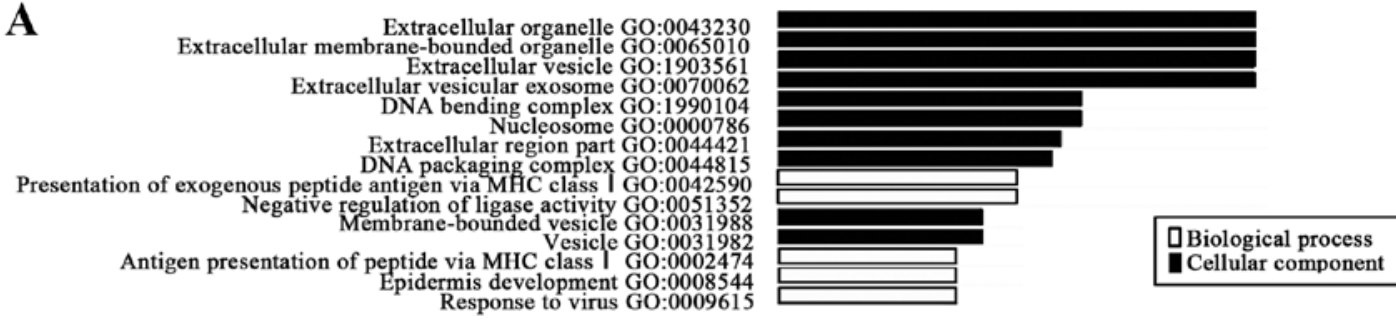
rmis development GO:00085

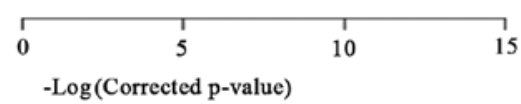

B

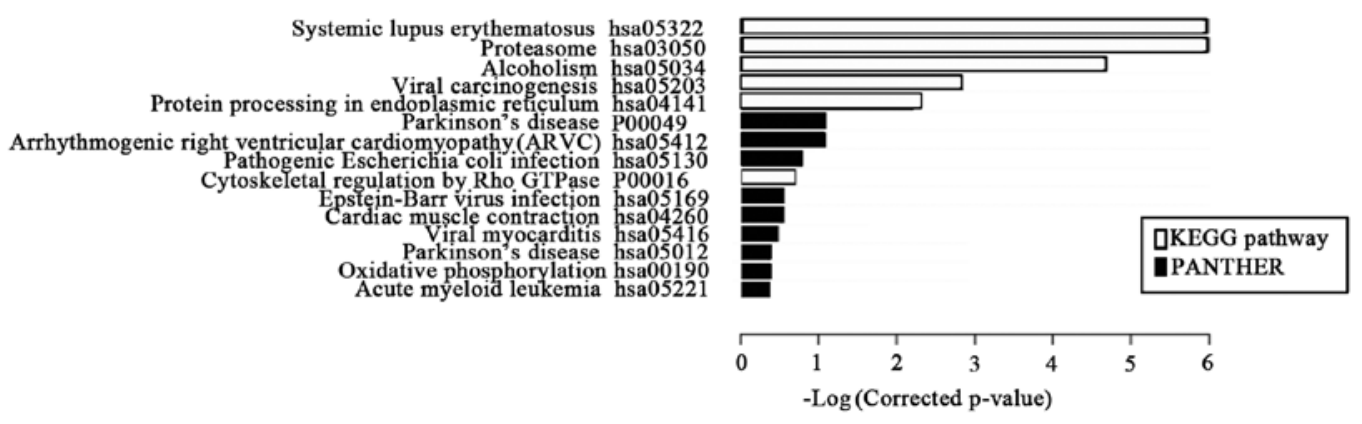

Figure 2. GO and pathway analysis of lncRNA co-expressed mRNAs. (A) The top 15 significantly enriched GO categories. The white bars represent biological processess and the black bars represent cellular components. (B) The top 15 pathway analysis of differentially expressed mRNAs. The white bars indicate significantly differential KEGG pathways and the black bars indicate pathways from PANTHER.

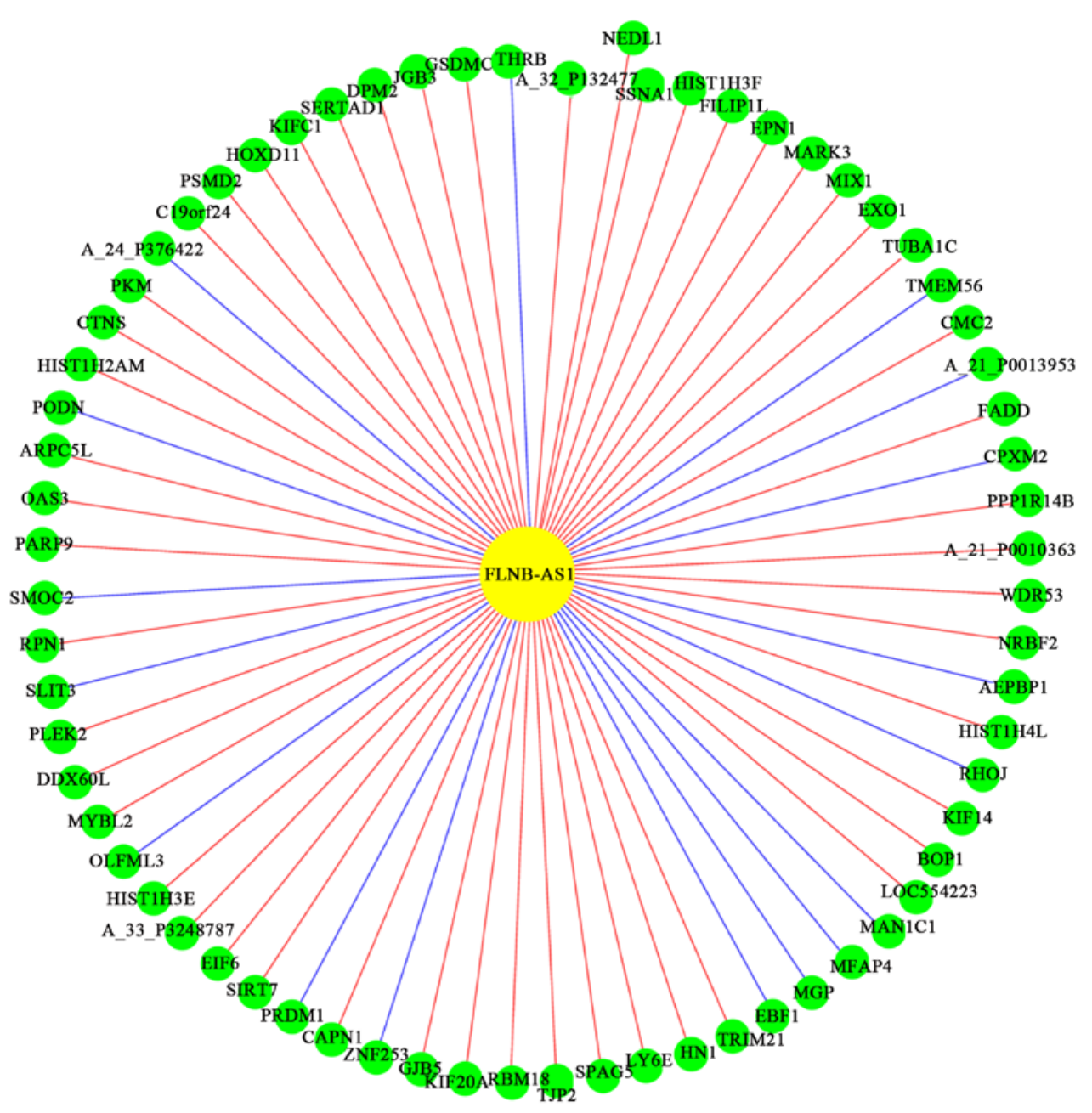

Figure 3. Co-expression network between the lncRNA (FLNB-AS1) and mRNAs in VSCC tissues with NTs. The green circles represent mRNAs. Red lines represent a positive interaction between transcripts and blue lines a negative interaction. 
A

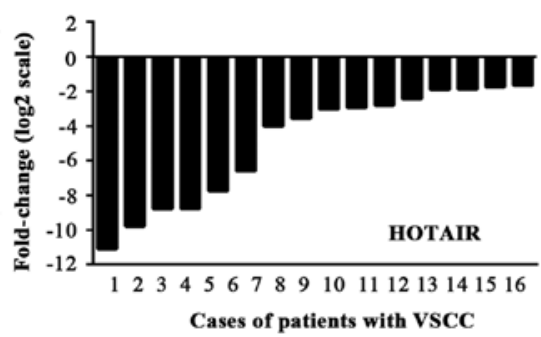

C

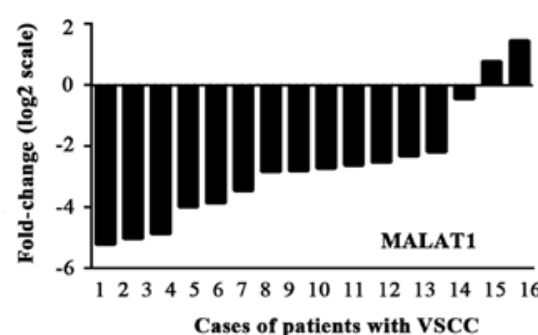

E

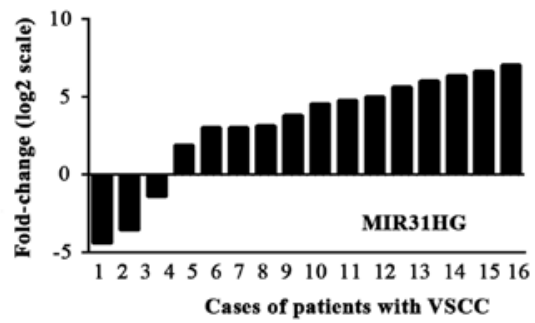

G

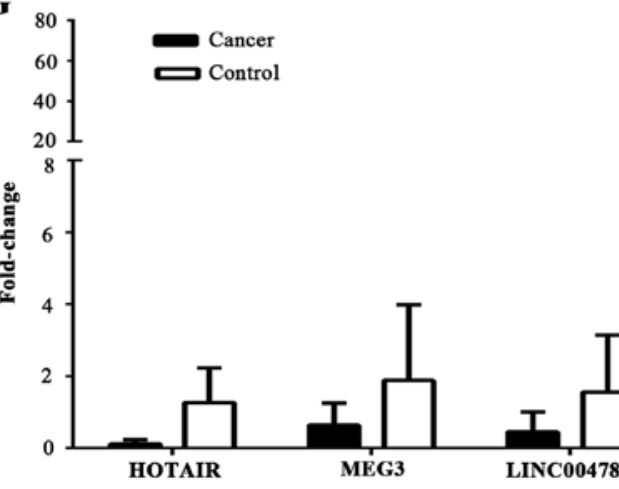

B

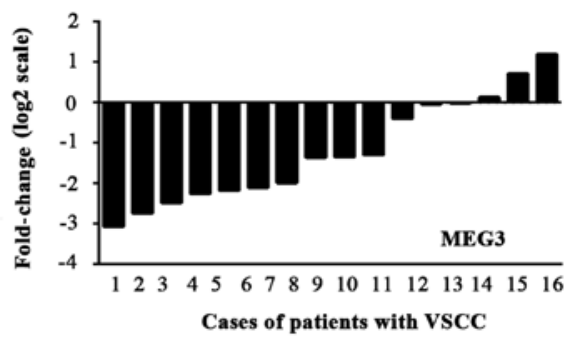

D

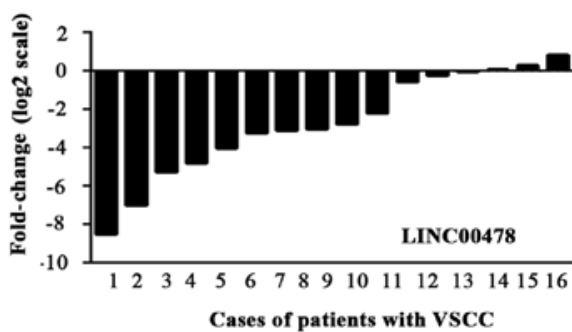

F

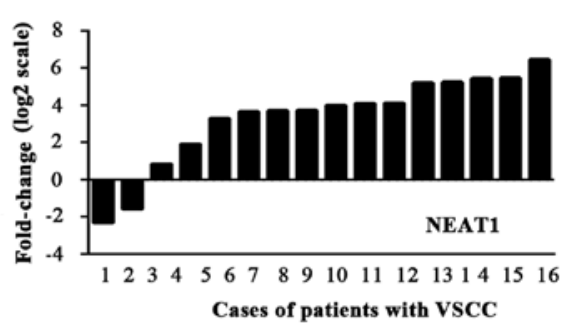

Figure 4. Validation of the differential expression of candidate lncRNAs using RT-PCR. (A-F) Expression of HOTAIR, MEG3, LINC00478, MALAT1, MIR31HG and NEAT1 in VSCC patients. (G) The expression levels of lncRNAs were quantified using RT-PCR in all samples.

with extracellular organelles (Fig. 2), but none were linked with molecular function on GO analysis. Pathway analysis indicated that 38 pathways corresponded to the most significant differential pathways.

Overview of the co-expression network. In order to verify the correlation between differentially expressed lncRNAs and mRNAs, a coding/non-coding gene co-expression network was constructed in VSCC patients compared with the control group. The co-network showed that one lncRNA correlated with multiple mRNAs and reciprocally. According to the network, we found that IncRNA, FLNB-AS1, had the most correlative mRNAs such as HOXD11 and PKM (Fig. 3). Therefore, it was proposed that the expression profile of IncRNAs and mRNAs was significantly correlated.

Real-time PCR validation of several differentially expressed lncRNAs. According to fold difference, one upregulated

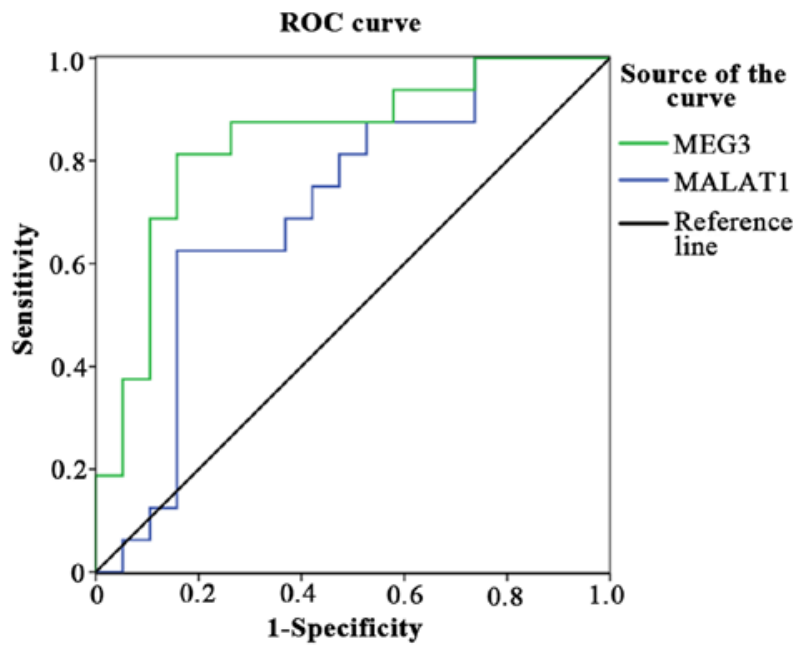

Figure 5. ROC curves of MEG3 and MALAT1. The green line represents MEG3 and the blue one represents MALAT1. The black line is a reference line. 
lncRNA (NEAT1) was initially selected and five downregulated lncRNAs (MIR31HG, MALAT1 HOTAIR, LINC00478 and MEG3) were also selected for verification of expression in two sets of tissue samples. A general consistency between the microarray and real-time PCR showed that the six selected lncRNAs were dysregulated between VSCC tissues and the control group (Fig. 4). The levels of HOTAIR, MALAT1, MEG3 and LINC00478 $(p=0.000, p=0.001, p=0.023$ and $p=0.009$, respectively) expression were decreased and NEAT1 was upregulated as indicated by the microarray analysis $(\mathrm{p}=0.002)$; whereas high expression of MIR31HG in the 35 samples, was detected, in opposition to the microarray data $(\mathrm{p}=0.007)$.

Evaluation of MEG3 and MALAT1 as diagnostic biomarkers for VSCC. We constructed a receiver operating characteristic (ROC) curve to evaluate the diagnostic value of our findings. Both MEG3 and MALAT1 were downregulated in the VSCC samples. Among them, MEG3 was only detected in $19 \%(3 / 16)$ of the tumor samples, meanwhile, MALAT1 was found downregulated in $87.5 \%(14 / 16)$ of the VSCC samples. Furthermore, the areas under the ROC curve (AUCs) were 0.707 and 0.839 , respectively ( $<<0.05)$ (Fig. 5).

Correlation between NEAT1, LINC00478 and MIR31HG expression and clinicopathological features in VSCC patients. Lymphatic metastasis is the important measure of prognosis. As shown in Table I, we found that the level of NEAT1 expression in VSCC tissues was associated with lymph node metastasis $(\mathrm{p}=0.002)$. We also observed that there was a statistical relationship between LINC00478 and MIR31HG expression and tumor differentiation $(\mathrm{p}=0.003, \mathrm{p}=0.007$ respectively). However, no significant correlations were found between lncRNAs levels and other clinicopathological factors studied, including the International Federation of Gynecology and Obstetrics (FIGO) stage.

\section{Discussion}

Recent research has demonstrated the key role of IncRNAs in regulating embryogenesis and gene expression, and lncRNAs have emerged as drivers of tumor suppressive and oncogenic functions in various human solid tumors (13-16). Our results revealed that aberrantly expressed lncRNAs may be a factor in VSCC pathogenesis, potentially providing new biomarkers and therapeutic targets for VSCC.

VSCC is a rare malignant tumor, however, its morbidity and mortality rates have risen in the past few decades. It is generally believed that VSCC has two etiological pathways, i.e., a high-risk human papillomavirus (HPV)-dependent route and genetic alterations such as p53 mutations and silencing of p16INK4a $(17,18)$.

Homeobox transcript antisense RNA (HOTAIR) is a well-known lncRNA transcribed from the HOXC cluster located on chromosome 12q13.3 (19). It has been proven that HOTAIR is involved in cancer progression and prognosis and may be an important target for cancer diagnosis and therapy (20-22). According to microarray analysis, the present study revealed that the level of HOTAIR expression was decreased in $75 \%(3 / 4)$ of the paired samples of tumor tissues. According to Sharma et al, there is a significant linear trend towards progressive HOTAIR downregulation among HPV-negative controls, HPV-positive non-malignant tumors and cervical carcinoma samples. They speculated that HOTAIR could be a potential target of E7, in HPV16-related cervical cancers (23).

Metastasis-associated lung adenocarcinoma transcript 1 (MALAT1) is another long lncRNA correlated with HPV. Jiang et al found that MALAT1 was expressed in HPV-positive cervical squamous cells, but not in HPV-negative normal cervical squamous cells, which suggests that HPV correlates with MALAT1 deregulation in cervical cancer (24). Various previous studies have indicated that HOTAIR and MALAT1 are overexpressed in tumor tissues compared with the normal tissues (25-28). It was thought that HOTAIR and MALAT1 contribute to carcinogenesis in most tumors whereas other studies proved the role of HOTAIR and MALAT1 as tumor suppressors. HOTAIR expression was found to be increased in breast cancer patients; nonetheless, knockdown of HOTAIR could inhibit invasion (15). Lu et al found that breast cancer patients with HOTAIR high-expression had lower risk of recurrence and poor prognosis (29). It has also been demonstrated that the level of expression of MALAT1 in gliomas was lower than that in normal tissues (30). These discrepant results suggest that lncRNAs can differentially express depending on the cancer types and that lncRNAs have active roles in tumorigenesis. Moreover, our data revealed the opposite results when compared to many other studies, namely that HOTAIR and MALAT1 were downregulated in VSCC tissues. Firstly, we inferred that HPV infection may be involved. Both HOTAIR and MALAT1 are correlated with HPV. Unfortunately, some of the patients we evaluated refused HPV examination and we were unable to determine the number of patients originally infected with HPV. HPV infection is not the most crucial factor in the level of expression of lncRNAs, since it is irrelevant in other types of tumors. However, HPV examination is still necessary for VSCC patients. In addition, it is meaningful that HPV infection be considered in lncRNA expression analysis as it is a useful factor. We hypothesized that lncRNAs such as HOTAIR and MALAT1 are expressed differentially in HPV-positive and HPV-negative VSCC samples and that these two lncRNAs may be involved in VSCC tumorigenesis by targeting HPV. Secondly, the number of cases is one limitation in our research. Thus, larger-scale studies are needed to validate our findings for further research.

The TP53-associated route and other genetic changes, such as CDKN2A (p16), also play a key role in the development of VSCC. Genetic mutations in TP53 have been detected in HPV-negative VSCCs. They may represent early changes in HPV-independent vulvar carcinogenesis (31). Expression of p53 was significantly increased in VSCC patient samples compared with the control group (32).

Maternally expressed gene 3 (MEG3) is a lncRNA which has been recently associated with $\mathrm{p} 53$. Lu et al found that $\mathrm{p} 53$ protein levels are affected by MEG3 overexpression in vitro. MEG3 was significantly downregulated in non-small cell lung cancer (NSCLC) tissues that may be affected by DNA methylation, and it partially regulated NSCLC cell proliferation and apoptosis via the activation of p53 (33). Zhu et al found that the level of MEG3 expression was reduced in hepatoma samples 
compared with adjacent non-tumor samples and interacts with the p53 DNA binding domain. Moreover, various p53 target genes were found to be deregulated after overexpression of MEG3 in hepatoma cells (34). Our data showed that MEG3 was downregulated in the VSCC patient samples. We speculate that MEG3 may operate as a tumor-suppressor in VSCC, whose mechanism may involve activation of p53.

Epigenetic silencing of p16 is also an early and important event in vulvar neoplasia (19). Montes et al showed that the lncRNA, MIR31HG, is upregulated in oncogene-induced senescence (OIS) which is considered an important mechanism of tumor suppression. They also found that knockdown of MIR31HG promoted a strong p16INK4A-dependent senescence phenotype. These results suggest that MIR31HG may repress p16INK4A expression in human cancer (35). MIR31HG is underactive in most cancers, but in lung and breast cancers, it appears to be upregulated by an, as yet, unknown mechanism $(36,37)$. According to the microarray analysis, the present study revealed that the level of MIR31HG expression was decreased in $75 \%$ (3/4) of the paired samples of tumor tissues while it was upregulated in VSCC samples by RT-PCR. These findings imply that the microarray profiling results that are based on a small sample size may not entirely be reliable and need to be further verified by RT-PCR analysis using a greater number of samples.

The lncRNA MIR31HG is also correlated with poor prognosis and may be an important target for cancer diagnosis and therapy in gastric cancer (38). According to the present study, we found that the increased level of MIR31HG expression in VSCC was significantly associated with tumor differentitation (Table I), suggesting that MIR31HG may play a critical role, not only in the tumorigenesis of VSCC, but also in its prognosis. Patients with higher expression levels of MIR31HG may have poorer prognosis. However, not all patients who were diagnosed with VSCC within the last three years at our institution underwent surgery, and only two patients died of VSCC, therefore, we were unable to obtain reliable survival analysis.

Nuclear paraspeckle assembly transcript 1 (NEAT1) is a prognostic biomarker as well as MIR31HG (39). NEAT1 was upregulated in 75\% (3/4) of VSCC tissues in accordance with the results of the microarray profile (Fig. 4). According to the study of Li et al, NEAT1 may play an oncogenic role in colorectal cancer which is involved in differentiation, invasion and metastasis (40). Our results revealed a greater association between NEAT1 expression and lymph node metastasis (Table I). Lymphatic metastasis usually indicates poor prognosis, suggesting that NEAT1 may play an important role in the prognosis of VSCC.

lncRNAs are emerging as biomarkers based upon ncRNA biology. Similar to microRNAs, various lncRNAs have been found in both urine and plasma, which can be easily obtained and provide for readily-available and stable diagnostics in order to detect cancers and cancer subtypes $(41,42)$.

IncRNAs, such as MEG3 and MALAT1, have been shown to be potential biomarkers in other types of cancers. However, there are some critical issues that still need to be resolved before they can be applied clinically. Most recent studies have been performed using only small-scale samples. Therefore, further large-scale studies are needed to validate their find- ings. In addition, since conventional methods such as RNA extraction take too long and the quality of lncRNAs can be influenced by multiple factors during the analytical process, more stable platforms and more rapid analytical methods are required. Finally, the expression of lncRNAs could be confounded by multiple factors in tumorigenesis procedures such as pathology. Thus, our results must be validated in larger independent cohorts to confirm that lncRNAs can be used as reliable biomarkers for cancer detection.

The present study had several limitations including its small sample size. Although our results suggested that several IncRNAs were potentially important biomarkers in VSCC, we collected only 16 samples from VSCC patients. Thus, our results require validation using larger prospective studies involving a larger number of samples. Furthermore, not all patients who were diagnosed with VSCC within the last three years underwent surgery; some were lost in the follow-up and only two patients died from VSCC. Thus, it was difficult to arrive at a reliable survival curve due to the small number of cases available. In addition, there were no stage IV patients in our cases since most patients with stage IV chose not to undergo surgery. Therefore, future experiments targeting these aspects are needed to verify our results.

In conclusion, the present study was the first to determine global aberrant lncRNA expression in VSCC tissues compared with the adjacent non-tumorous tissues using microarray analysis. We also identified a panel of dysregulated lncRNAs that may be potential biomarkers as they were also correlated with VSCC carcinogenesis. These dysregulated lncRNAs may be VSCC-specific lncRNAs and may form the basis for future diagnostic, therapeutic and functional research on VSCC.

\section{References}

1. Woelber L, Kock L, Gieseking F, Petersen C, Trillsch F, Choschzick M, Jaenicke F and Mahner S: Clinical management of primary vulvar cancer. Eur J Cancer 47: 2315-2321, 2011.

2. Bertone P, Stolc V, Royce TE, Rozowsky JS, Urban AE, Zhu X, Rinn JL, Tongprasit W, Samanta M, Weissman S, et al: Global identification of human transcribed sequences with genome tiling arrays. Science 306: 2242-2246, 2004.

3. Derrien T, Johnson R, Bussotti G, Tanzer A, Djebali S, Tilgner H, Guernec G, Martin D, Merkel A, Knowles DG, et al: The GENCODE $v 7$ catalog of human long noncoding RNAs: Analysis of their gene structure, evolution, and expression. Genome Res 22: 1775-1789, 2012.

4. Geisler S and Coller J: RNA in unexpected places: Long noncoding RNA functions in diverse cellular contexts. Nat Rev Mol Cell Biol 14: 699-712, 2013.

5. Roberts TC, Morris KV and Weinberg MS: Perspectives on the mechanism of transcriptional regulation by long non-coding RNAs. Epigenetics 9: 13-20, 2014.

6. Wang KC and Chang HY: Molecular mechanisms of long noncoding RNAs. Mol Cell 43: 904-914, 2011.

7. Guttman M, Amit I, Garber M, French C, Lin MF, Feldser D, Huarte M, Zuk O, Carey BW, Cassady JP, et al: Chromatin signature reveals over a thousand highly conserved large non-coding RNAs in mammals. Nature 458: 223-227, 2009.

8. Mercer TR, Dinger ME and Mattick JS: Long non-coding RNAs: Insights into functions. Nat Rev Genet 10: 155-159, 2009.

9. Huarte $M$ and Rinn JL: Large non-coding RNAs: Missing links in cancer? Hum Mol Genet 19: R152-R161, 2010.

10. Takahashi K, Yan I, Haga $\mathrm{H}$ and Patel T: Long noncoding RNA in liver diseases. Hepatology 60: 744-753, 2014.

11. Song H, Sun W, Ye G, Ding X, Liu Z, Zhang S, Xia T, Xiao B, $\mathrm{Xi} Y$ and Guo J: Long non-coding RNA expression profile in human gastric cancer and its clinical significances. J Transl Med 11: 225, 2013. 
12. Livak KJ and Schmittgen TD: Analysis of relative gene expression data using real-time quantitative PCR and the $2^{-\Delta \Delta C_{\mathrm{T}}}$ method. Methods 25: 402-408, 2001.

13. Prensner JR and Chinnaiyan AM: The emergence of lncRNAs in cancer biology. Cancer Discov 1: 391-407, 2011.

14. Tsai MC, Spitale RC and Chang HY: Long intergenic noncoding RNAs: New links in cancer progression. Cancer Res 71: 3-7, 2011.

15. Gupta RA, Shah N, Wang KC, Kim J, Horlings HM, Wong DJ, Tsai MC, Hung T, Argani P, Rinn JL, et al: Long non-coding RNA HOTAIR reprograms chromatin state to promote cancer metastasis. Nature 464: 1071-1076, 2010.

16. Ponting CP, Oliver PL and Reik W: Evolution and functions of long noncoding RNAs. Cell 136: 629-641, 2009.

17. Trietsch MD, Nooij LS, Gaarenstroom KN and van Poelgeest MI: Genetic and epigenetic changes in vulvar squamous cell carcinoma and its precursor lesions: A review of the current literature. Gynecol Oncol 136: 143-157, 2015.

18. Rinn JL, Kertesz M, Wang JK, Squazzo SL, Xu X, Brugmann SA Goodnough LH, Helms JA, Farnham PJ, Segal E, et al: Functional demarcation of active and silent chromatin domains in human HOX loci by noncoding RNAs. Cell 129: 1311-1323, 2007.

19. Gasco M, Sullivan A, Repellin C, Brooks L, Farrell PJ, Tidy JA, Dunne B, Gusterson B, Evans DJ and Crook T: Coincident inactivation of $14-3-3 \sigma$ and $16^{\mathrm{INK} 4 \mathrm{a}}$ is an early event in vulval squamous neoplasia. Oncogene 21: 1876-1881, 2002.

20. Lv XB, Lian GY, Wang HR, Song E, Yao H and Wang MH: Long noncoding RNA HOTAIR is a prognostic marker for esophageal squamous cell carcinoma progression and survival. PLoS One 8: e63516, 2013.

21. Chen FJ, Sun M, Li SQL, Wu QQ, Ji L, Liu ZL, Zhou GZ, Cao G, Jin L, Xie HW, et al: Upregulation of the long non-coding RNA HOTAIR promotes esophageal squamous cell carcinoma metastasis and poor prognosis. Mol Carcinog 52: 908-915, 2013.

22. Ge XS, Ma HJ, Zheng XH, Ruan HL, Liao XY, Xue WQ, Chen YB, Zhang Y and Jia WH: HOTAIR, a prognostic factor in esophageal squamous cell carcinoma, inhibits WIF-1 expression and activates Wnt pathway. Cancer Sci 104: 1675-1682, 2013.

23. Sharma S, Mandal P, Sadhukhan T, Roy Chowdhury R, Ranjan Mondal N, Chakravarty B, Chatterjee T, Roy S and Sengupta S: Bridging links between long noncoding RNA HOTAIR and HPV oncoprotein E7 in cervical cancer pathogenesis. Sci Rep 5: 11724, 2015.

24. Jiang Y, Li Y, Fang S, Jiang B, Qin C, Xie P, Zhou G and Li G: The role of MALAT1 correlates with HPV in cervical cancer Oncol Lett 7: 2135-2141, 2014.

25. Li D, Feng J, Wu T, Wang Y, Sun Y, Ren J and Liu M: Long intergenic noncoding RNA HOTAIR is overexpressed and regulates PTEN methylation in laryngeal squamous cell carcinoma. Am J Pathol 182: 64-70, 2013.

26. Wu ZH, Wang XL, Tang HM, Jiang T, Chen J, Lu S, Qiu GQ, Peng ZH and Yan DW: Long non-coding RNA HOTAIR is a powerful predictor of metastasis and poor prognosis and is associated with epithelial-mesenchymal transition in colon cancer. Oncol Rep 32: 395-402, 2014.

27. Ishibashi M, Kogo R, Shibata K, Sawada G, Takahashi Y, Kurashige J, Akiyoshi S, Sasaki S, Iwaya T, Sudo T, et al: Clinical significance of the expression of long non-coding RNA HOTAIR in primary hepatocellular carcinoma. Oncol Rep 29: 946-950, 2013.
28. Li X, Wu Z, Mei Q, Li X, Guo M, Fu X and Han W: Long non-coding RNA HOTAIR, a driver of malignancy, predicts negative prognosis and exhibits oncogenic activity in oesophageal squamous cell carcinoma. Br J Cancer 109: 2266-2278, 2013.

29. Lu L, Zhu G, Zhang C, Deng Q, Katsaros D, Mayne ST, Risch HA, Mu L, Canuto EM, Gregori G, et al: Association of large noncoding RNA HOTAIR expression and its downstream intergenic $\mathrm{CpG}$ island methylation with survival in breast cancer. Breast Cancer Res Treat 136: 875-883, 2012.

30. Han Y, Wu Z, Wu T, Huang Y, Cheng Z, Li X, Sun T, Xie X, Zhou Y and Du Z: Tumor-suppressive function of long noncoding RNA MALAT1 in glioma cells by downregulation of MMP2 and inactivation of ERK/MAPK signaling. Cell Death Dis 7: e2123, 2016.

31. Del Pino M, Rodriguez-Carunchio L and Ordi J: Pathways of vulvar intraepithelial neoplasia and squamous cell carcinoma. Histopathology 62: 161-175, 2013.

32. Sadalla JC, Lourenço SV, Sotto MN, Baracat EC and Carvalho JP: Claudin and p53 expression in vulvar lichen sclerosus and squamous-cell carcinoma. J Clin Pathol 64: 853-857, 2011.

33. Lu KH, Li W, Liu XH, Sun M, Zhang ML, Wu WQ, Xie WP, Hou YY, Lu KH and Li W: Long non-coding RNA MEG3 inhibits NSCLC cells proliferation and induces apoptosis by affecting p53 expression. BMC Cancer 13: 461, 2013.

34. Zhu J, Liu S, Ye F, Shen Y, Tie Y, Zhu J, Wei L, Jin Y, Fu H, Wu Y, et al: Long noncoding RNA MEG3 interacts with p53 protein and regulates partial p53 target genes in hepatoma cells. PLoS One 10: e0139790, 2015

35. Montes M, Nielsen MM, Maglieri G, Jacobsen A, Højfeldt J, Agrawal-Singh S, Hansen K, Helin K, van de Werken HJ Pedersen JS, et al: The IncRNA MIR3IHG regulates p16 ${ }^{\mathrm{INK} 4 \mathrm{~A}}$ expression to modulate senescence. Nat Commun 6: 6967, 2015.

36. Shi Y, Lu J, Zhou J, Tan X, He Y, Ding J, Tian Y, Wang L and Wang K: Long non-coding RNA Loc554202 regulates proliferation and migration in breast cancer cells. Biochem Biophys Res Commun 446: 448-453, 2014.

37. Xi S, Yang M, Tao Y, Xu H, Shan J, Inchauste S, Zhang M, Mercedes L, Hong JA, Rao M, et al: Cigarette smoke induces C/EBP- $\beta$-mediated activation of miR-31 in normal human respiratory epithelia and lung cancer cells. PLoS One 5: e13764, 2010.

38. Nie FQ, Ma S, Xie M, Liu YW, De W and Liu XH: Decreased long noncoding RNA MIR31HG is correlated with poor prognosis and contributes to cell proliferation in gastric cancer. Tumour Biol: Dec 21, 2015 (Epub ahead of print).

39. Wu Y, Yang L, Zhao J, Li C, Nie J, Liu F, Zhuo C, Zheng Y, Li B, Wang Z, et al: Nuclear-enriched abundant transcript 1 as a diagnostic and prognostic biomarker in colorectal cancer. Mol Cancer 14: 191, 2015.

40. Li Y, Li Y, Chen W, He F, Tan Z, Zheng J, Wang W, Zhao Q and Li J: NEAT expression is associated with tumor recurrence and unfavorable prognosis in colorectal cancer. Oncotarget 6: 27641-27650, 2015.

41. Huang X, Yuan T, Tschannen M, Sun Z, Jacob H, Du M, Liang M, Dittmar RL, Liu Y, Liang M, et al: Characterization of human plasma-derived exosomal RNAs by deep sequencing. BMC Genomics 14: 319, 2013.

42. Lee GL, Dobi A and Srivastava S: Prostate cancer: Diagnostic performance of the $P C A 3$ urine test. Nat Rev Urol 8: 123-124, 2011. 